\section{Wolfgang G. Stock - Würdigung seines Werkes durch einen Weggefährten}

https://doi.org/10.1515/iwp-2019-2026

Alles nahm an der Karl-Franzens-Universität Graz seinen Anfang, wo Wolfgang G. Stock maßgeblich am Aufbau der Dokumentation zur Österreichischen Philosophie unter der Leitung von Prof. Rudolf Haller mitwirkte und wo er sich schließlich auch habilitierte. Mit der Gründung des Instituts für Informationswissenschaft an der Sozial- und Wirtschaftswissenschaftlichen Fakultät der Uni Graz war eine weitere Zusammenarbeit vorprogrammiert. So hielt Wolfgang G. Stock vom Wintersemester 1988/89 bis zum Wintersemester 1992/93 die Vorlesung „Dokumentationsmethoden“, in den beiden darauf folgenden Wintersemestern die Vorlesung „Wirtschaftsinformationen aus Datenbanken“. Während dieser Zeit hatte der Autor dieses Beitrags das Vergnügen, Wolfgang G. Stock kennenzulernen. Aus der Bekanntschaft entwickelte sich schließlich eine langjährige Freundschaft, die bis jetzt andauert.

Diese wurde von einer Reihe von wissenschaftlichen „Projekten“ begleitet. In die Zeit, in der Wolfgang G. Stock eine informationswissenschaftliche Professur an der Fachhochschule Köln innehatte, fällt eine gemeinsame Lehrveranstaltung mit sechs Studierenden der Uni Graz und acht Studierenden der Fachhochschule Köln, in der die bedeutendsten Zeitschriften unseres Faches einer szientometrischen Analyse unterzogen wurden. Konkret wurde für 40 internationale und zehn deutschsprachige Zeitschriften der Bibliotheks- und Informationswissenschaft eine Zitatanalyse durchgeführt. Insgesamt wurden ca. 6.200 Artikel mit ungefähr 90.000 Referenzen analysiert. Zusätzlich wurden im Rahmen einer E-Mail-Erhebung Fachexperten im deutschsprachigen Raum über ihre Lese-, Nutzungsund Publikationspräferenzen befragt (Grazia Colonia, 2002). Bemerkenswert an dieser Studie ist, dass die Ergebnisse nicht nur in deutschsprachigen, sondern auch in einer der führenden internationalen Fachzeitschriften (Journal of the American Society for Information Science and Technology) publiziert werden konnten.

Die Berufung von Wolfgang G. Stock an die Abteilung für Informationswissenschaft der Heinrich-Heine-Universität Düsseldorf verstärkte die Zusammenarbeit weiter. Unter anderem wurde das Zeitschriftenprojekt fortgesetzt, dessen Ergebnisse in den angesehenen Zeitschriften „Library Collections, Acquisitions, and Technical Services“ und „Journal of Documentation“ veröffentlicht werden

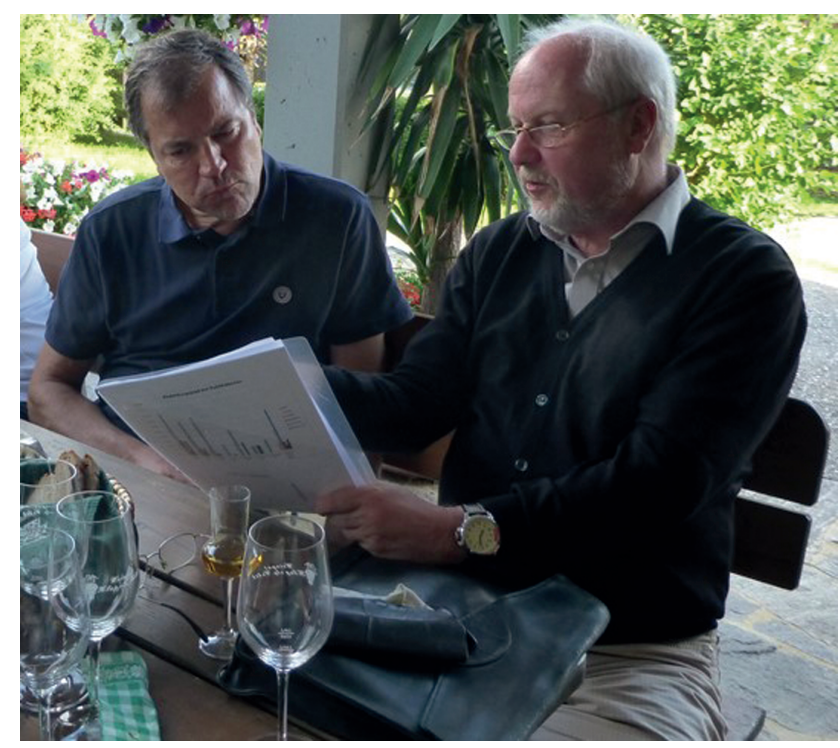

Abbildung 1: Prof. Rudolf Stock und der Autor. (Foto: Christian Schlögl)

konnten. Auf institutioneller Ebene wurde die Zusammenarbeit zwischen den Instituten durch Erasmusverträge untermauert. Eine der jüngsten Kooperationen war die Herausgabe von zwei Schwerpunktheften der Zeitschrift „Information - Wissenschaft \& Praxis (IWP)“ (Heft 5-6/ 2017 und Heft 1/2018) zu den Forschungsthemen der Düsseldorfer und Grazer Informationswissenschaft.

Im Folgenden möchte ich den Versuch unternehmen, auf die wissenschaftlichen Leistungen von Wolfgang G. Stock kurz einzugehen. Aus der Sicht eines Wissenschaftlers sind dabei insbesondere Forschung und Lehre zu betrachten:

Wolfgang G. Stock ist ein leidenschaftlicher Forscher. Besonders bemerkenswert finde ich seine hohe Forschungsproduktivität, die bisher in mehr als 300 Publikationen ihren Niederschlag fand. Viele davon erschienen in internationalen und qualitativ hochwertigen Fachzeitschriften. Beispielsweise kann Wolfgang G. Stock auf um die 50 Veröffentlichungen im Web of Science verweisen. Darüber hinaus liegen auch mehrere Buchpublikationen von ihm vor. Es sind dies u.a. Informationswirtschaft (2018), Handbook of Information Science (2015), Information Markets (2011), Wissensrepräsentation: Informationen auswerten und bereitstellen (2008) sowie Information Retrieval: Informationen suchen und finden (2007). Zudem ist er seit 2009 Herausgeber der von DeGruyter Saur verlegten Schriftenreihe „Knowledge and Information. Studies in Information Science“ (https://www.degruyter.com/view/serial/39677). Die Publikationen von Wolfgang G. Stock wurden auch häufig zitiert, die Web of Science-Publikationen beispielsweise ca. 400 Mal (h-In- 
dex: > 10). In Google Scholar erhielt Wolfgang G. Stock sogar an die 3000 Zitate (h-Index: bei 30). Aus obigen Ausführungen geht klar hervor, dass Kollege Stock international einer der am stärksten wahrgenommenen Informationswissenschaftler aus den deutschsprachigen Ländern ist.

Bei seinen Forschungsinhalten ist Wolfgang G. Stock recht breit aufgestellt. Erwähnenswert ist, dass er auch neuere, innovative Themen aufgegriffen hat. Unter seinen aktuellen Forschungsschwerpunkten finden sich beispielsweise Inhalte wie informationelle Städte, Social Media und Social Live-Streaming.

In der Lehre pflegte Wolfgang G. Stock einen kollegialen Umgang mit seinen Studierenden. Trotzdem hat er von seinen Studierenden auch viel verlangt. Besonders gut schaffte er den Spagat zwischen Praxis- und Forschungsbezug. All dies führte zu einem „Wiederaufblühen" der informationswissenschaftlichen Studiengänge an der Heinrich-Heine-Universität. Zuletzt waren dies die beiden Bachelorstudiengänge „Informationswissenschaft und Sprachtechnologie“ und „Informationswissenschaft als Ergänzungsfach“ sowie der Masterstudiengang „Informationswissenschaft und Sprachtechnologie“. Diese erfreuten sich bei den Studierenden großer Beliebtheit, u.a. auch deshalb, weil die Absolventinnen und Absolventen gute Jobchancen vorfanden. Es ist daher sehr schade, wenn diese Studiengänge in der jetzigen Form mit der Pensionierung von Wolfgang G. Stock auslaufen. Der Um- stand, dass die Professur aus der Informationswissenschaft nicht mehr nachbesetzt wird, zeigt leider auch, dass die Informationswissenschaft selbst dann nicht von einer Schließung ausgenommen ist, wenn sie in Forschung und Lehre sehr erfolgreich war.

Bei der großen Aktivitätenfülle von Wolfgang G. Stock ist die Diagnose „Pensionsschock“ für den neuen Lebensabschnitt nicht völlig auszuschließen. Um die Gefahr seines tatsächlichen Eintretens etwas zu reduzieren, wird Wolfgang G. Stock im kommenden Wintersemester mit einer Gastprofessur an der Universität Graz betraut werden.

Abschließend wünscht der Verfasser dieses Beitrag Wolfgang G. Stock für die neue Lebensphase alles Gute und freut sich auf viele weitere anregende Fachdiskussionen.

\section{Quellen:}

Gracia Colonia (2002). Informationswissenschaftliche Zeitschriften in szientometrischer Analyse. Kölner Arbeitspapiere zur Bibliotheks- und Informationswissenschaft. Band 33. Fachhochschule Köln, Fachbereich Informationswissenschaft.

ao. Univ. Prof. Dr. Christian Schlögl Universität Graz 